\title{
Editorial
}

\section{Role of PET/CT in Gastrointestinal Stromal Tumors}

\author{
El-Hennawy $\mathbf{G}^{\mathbf{1}}$. Moustafa, $\mathbf{H}^{2}$. \\ ${ }^{1}$ Nuclear Medicine Unit National Cancer Institute, Cairo University, ${ }^{2}$ Nuclear Medicine \& \\ Radiation Oncology Center, Cairo University, Egypt
}

The first accurate description of mesenchymal neoplasms of the gastrointestinal tract (GIT) was in 1941.

With the advent of immuno-histochemical analysis a definition of a new entity among gastrointestinal mesenchymal tumors called the gastrointestinal stromal tumors (GISTs); which particularly express the kit (CD117) protein; a growth factor trans-membrane receptor with tyrosine kinase activity ${ }^{(1)}$.

Gastrointestinal stromal tumors (GISTs) are the most frequent mesenchymal tumors of the gastrointestinal $\operatorname{tract}^{(2)}$. GISTs are usually found in the stomach or small intestine but can occur anywhere along the gastrointestinal tract and rarely have extra-gastrointestinal involvement. Approximately $50-70 \%$ of GISTs originate in the stomach. The small intestine is the second most common location, with $20-30 \%$ of GISTs arising from the jejuno-ileum. Less frequent sites of occurrence include the colon and rectum $(5-15 \%)$ and esophagus $(<5 \%)$.
Primary pancreatic, omental, or mesenteric GISTs have been reported but are very rare ${ }^{(3)}$.

Surgery remains the mainstay of therapy for patients with primary GIST who do not have evidence of metastasis and should be the initial therapy if the tumor is technically resectable with acceptable risk of morbidity. Regarding patients with a positive microscopic margin on final pathologic analysis, the management is still not well defined. There is no evidence that those patients with complete resection of all macroscopic disease, but still have microscopically positive margins, need to undergo re-excision. Lymphadenectomy is usually unnecessary because lymph node metastases are rare with GIST and sarcomas in general ${ }^{(4)}$.

There are different schemes for classification of GISTs on the basis of tumor size and mitotic count into very low risk, low risk, intermediate risk, and high risk. Alternatively, they are classified 
according to site, size, and mitotic activity into 3 categories: benign, malignant, and uncertain or low malignant potential. Gastrointestinal stromal tumors are subclassified according to their cellular pattern into spindle, epithelioid, and mixed patterns. A final consensus on GISTs classification has not been achieved, and the biologic behavior is often uncertain. Whether borderline GISTs are precursors of malignant GISTs that accumulate genetic alteration during malignant transformation or whether they represent a biologically indolent and distinct subset of GISTs is still uncertain $^{(5)}$.

\section{Keywords;}

Corresponding Author: El-Hennawy, G.

The seventh edition of the UICC published at the beginning of 2010 included for the first time a classification and staging system for GIST. This represents a major step towards a more standardized surgical and oncological treatment for patients with GIST and, most importantly, may facilitate the establishment of a uniformly applicable follow-up program based on tumor stage. The TNM system has applied the same criteria used for risk. In particular and different from any previously proposed TNM classification for a malignant neoplasm, four $\mathrm{T}$-categories have been separated solely on the basis of tumor size and then combined with mitotic rate and tumor site to define a clinical UICC stage. Given the rarity of nodal metastasis in GISTs in general. The presence of either

\section{E mail: gihanelhennawy@gmail.com}

nodal or distant metastasis heralds a stage UICC IV ${ }^{(6)}$.

GISTs have a characteristic immuno histochemical profile that is useful for confirming a suspected diagnosis ${ }^{(7)}$. About 95\% are positive for KIT (CD117), 60\% to $70 \%$ for CD34, $30 \%$ to $40 \%$ for smooth muscle actin, $5 \%$ for $\mathrm{S}-100$ protein, $1 \%$ to $2 \%$ for desmin, and $1 \%$ to $2 \%$ for keratin $^{(8)}$.

In general, KIT staining in GISTs is strongly and diffusely positive, but it is not necessarily uniform across different regions of the tumor ${ }^{(8)}$.

The morphology and immuno phenotype are concordant as KIT is expressed in nearly all GISTs. This marker has been emphasized and is often used for diagnosis .However; about 5\% of GISTs are truly negative for detectable KIT 
expression; so-called "KIT-negative GISTs $^{(8)}$.

\section{Contrast enhanced CT:}

\section{(A) Initial Evaluation:}

Baseline CT should be performed with oral contrast administration to define bowel margins. More importantly, use of intravenous contrast is essential to observe the degree and pattern of enhancement and the tumor vessels. Despite the large size of some GISTs, clinical evidence of gastrointestinal obstruction is uncommon. Metastasis may occur through locoregional infiltration or a hematogenous route of spread, most often to the liver, omentum, and peritoneal cavity. Metastases can also be found in the soft tissues (such as the abdominal wall) and rarely in the lungs and pleura, bone, or lymph nodes ${ }^{(8)}$.

\section{(B) Assessment of Response:}

In the early stages of imatinib therapy, the decreases in tumor size may not parallel changes in tumor density, and patients may have substantial symptomatic improvement even in the absence of tumor shrinkage. Traditional tumor response criteria such as RECIST are based on unidimensional tumor size and do not take into account changes in tumor metabolism, tumor density, and decrease in the number of intra-tumoral vessels. All of these changes indicate response to TKI therapy in patients with GIST. Hence, response assessment according to RECIST is known to be insensitive in evaluating response to TKI therapy ${ }^{(9)}$.

Partial response is considered if the size is reduced by at least $30 \%$ on CT scans. It was subsequently found that many of these tumors may not change significantly in size or may even grow larger in response to Imatinib mesylate administration before GISTs start shrinking as they undergo cystic changes and changes in density. Stable disease according to $\mathrm{CT}$ criteria (i.e., no tumor growth) has been shown to be predictive of time- to-treatment failure, and patient outcomes seem to better correlate with clinical and radiologic responses to imatinib than PET-CT ${ }^{(10)}$.

CT plays an important role in showing tumor stability and identifying any true tumor progression that might signal the clonal emergence of resistance to imatinib. Tumor recurrence after surgical resection can be a metastasis or can occur at the site of primary disease $\mathrm{e}^{(8)}$.

$\mathrm{CT}$ is performed for surveillance of metastatic or recurrent disease, and abdominal/pelvic CT scans should be obtained every 3 to 6 months. For very low-risk GISTs, less-frequent follow-up is 
appropriate. When progression occurs, imaging frequency should be increased. Each treated lesion should be carefully analyzed for new intra-tumoral changes. $\mathrm{CT}$ is recommended within 3 months of initiating TKI therapy in patients with definitively un-resectable or metastatic disease $^{(8)}$.

\section{Role of FDG PET/CT in Gastro intestinal tumors (GIST)}

\section{Initial Staging:}

Initial results indicated that $\mathrm{CT}$ and $18 \mathrm{~F}-$ FDG PET have comparable sensitivity and positive predictive value in initial staging of malignant GISTs. Although CT scans had better anatomic resolution of the sites of lesions, the difference in performance between CT and 18F-FDG PET was not statistically significant $^{(11)}$. Choi et al. ${ }^{(12)}$ reported that $80 \%$ of lesions show a significant glucose uptake on pretreatment 18F -FDG-PET, hence existence of a baseline 18F-FDG-PET/CT as reference is essential. Consequently, when neo adjuvant imatinib therapy is considered, we believe that a baseline PET/CT is compulsory (12) .This has not been suggested in the NCCN guidelines ${ }^{(8)}$.

Initial reports suggest an impressive role for 18F-FDG PET in follow-up for assessment of therapy for GIST. Choi et al. (13) demonstrated that the overall disease status evaluated objectively according to changes in size, intensity of tracer uptake, and number of tumor nodules correlated best with the reduction of maximum SUV on 18F-FDG PET scans Figure (1).

The degree of uptake of 18F FDG in GIST is usually intense. The sensitivity and positive predictive value for the detection of GISTs by PET/CT have been described as 86 and $98 \%$, respectively, and falsenegative PET/CTs were mostly related to small lesions (14) .This described a correlation between histological grade of malignancy and 18F-FDG avidity by GIST, which seems to reflect the metabolic mitotic activity, so that PET may be a direct measure of tumor aggressiveness and thus of $\operatorname{prognosis}^{(15,16,17)}$.

The EORTC guidelines for the use of 18 FDG-PET as a biomarker of response suggest that a $25 \%$ reduction in SUV max should be considered as the threshold for partial metabolic response (PMR). We observed a similar prognostic value in this patient population when using that criterion $^{(18)}$. 


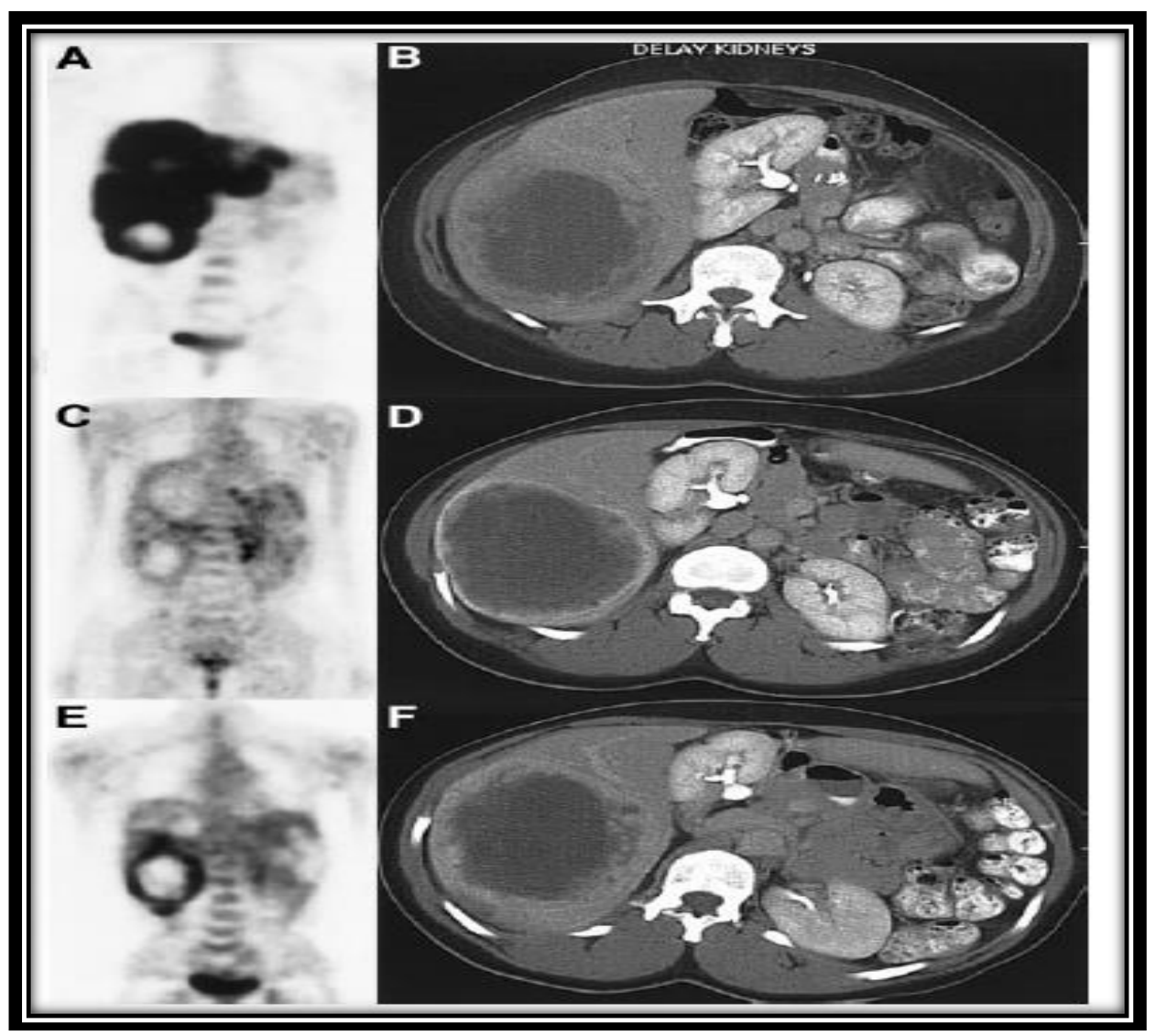

Figure (1).18F-FDG PET and CT scans of patient with metastatic GIST in abdomen and liver before therapy (A and B); at 12 mo of imatinibmesylate therapy (C and D); and at 13 mo after withdrawal of imatinibmesylate for $1 \mathrm{mo}$ ( $\mathrm{E}$ and F). Maximum SUV of abdominal mass changed from 10.1 (A) to 1.3 (C) to 4.5 (E), and tumor size in longest dimension changed from $10.9 \mathrm{~cm}(\mathrm{~B})$ to $11.3 \mathrm{~cm}(\mathrm{D})$ to $11.5 \mathrm{~cm}(\mathrm{~F}) .^{(13)}$

\section{Assessment of response in solid tracers}

1. Measurable target lesion is hottest single tumor lesion SUL of "maximal 1.2cm diameter volume ROI in tumor'" (SUL peak). SUL peak is at least 1.5 -fold greater than liver SUL mean +2SDs (in 3-cm spherical ROI in normal right lobe of liver). If liver is abnormal, primary tumor should have uptake $>2.0 \mathrm{X}$ SUL mean of blood pool in 1-cm-diameter ROI in descending thoracic aorta extended over 2cm z-axis.
2. Tumor with maximal SUL peak is assessed after treatment. Although typically this is in same region of tumor as that with highest SUL peak at baseline, it need not be.

3. Uptake measurements should be made for peak and maximal single-voxel tumor SUL. Other SUV metrics, including SUL mean at $50 \%$ or $70 \%$ of SUV peak, can be collected as exploratory data; TLG can be collected ideally on basis of voxels more intense than 2 SDs above liver mean SUL. 
4. These parameters can be recorded as exploratory data on up to 5 measurable target lesions, typically the 5 hottest lesions, which are typically the largest, and no more than 2 per organ. Tumor size of these lesions can be determined per RECIST $1.1^{(18)}$.

Normal liver SUL must be within $20 \%$ (and <0.3 SUL mean units) for baseline and follow-up study to be assessable. If liver is abnormal, blood-pool SUL must be within $20 \%$ (and <0.3 SUL mean units) for baseline and follow-up study to be assessable. Uptake time of baseline study and follow-up study 2 must be within 15 min of each other to be assessable. Typically, these are at mean of $60 \mathrm{~min}$ after injection but no less than 50 min after injection. Same scanner, or same scanner model at same site, injected dose, acquisition protocol (2-vs.3-dimensional), and software for reconstruction, should be used. Scanners should provide reproducible data and be properly calibrated $^{(18)}$.

\section{Complete Metabolic Response (CMR):}

Complete resolution of $18 \mathrm{~F}-\mathrm{FDG}$ uptake within measurable target lesion so that it is less than mean liver activity and indistinguishable from surrounding background blood-pool levels. Disappearance of all other lesions to background blood pool levels. Percentage decline in SUL should be recorded from measurable region, as well as (ideally) time in weeks after treatment was begun. No new 18F-FDG-avid lesions in pattern typical of cancer. If progression by RECIST, must verify with follow-up ${ }^{(18)}$.

\section{Partial Metabolic Response (PMR):}

Reduction of minimum of $30 \%$ in target measurable tumor 18F-FDG SUL peak. Absolute drop in SUL must be at least 0.8 SUL units, as well. Measurement is commonly in same lesion as baseline but can be another lesion if that lesion was previously present and is the most active lesion after treatment ${ }^{(18)}$.

ROI does not have to be in precisely same area as baseline scan, though typically it is. No increase, $>30 \%$ in SUL or size of target or non-target lesions (i.e., no PD by RECIST or IWC) (if PD anatomically, must verify with follow-up). Reduction in extent of tumor 18F-FDG uptake is not requirement for PMR. Percentage decline in SUL should be recorded, as well as (ideally) time in weeks after treatment was begun. No new lesions ${ }^{(18)}$.

\section{Stable Metabolic Disease (SMD):}

Not CMR, PMR, or PMD. SUL peak in metabolic target lesion should be recorded, as well as (ideally) time from start of most recent therapy, in weeks ${ }^{(18)}$.

\section{Progressive Metabolic Disease (PMD):}


$>30 \%$ increase in $18 \mathrm{~F}-\mathrm{FDG}$ SUL peak, with $>0.8$ SUL unit increase in tumor SUV peak from baseline scan in pattern typical of tumor and not of infection/treatment effect or visible increase in extent of $18 \mathrm{~F}$ FDG tumor uptake (75\% in TLG volume with no decline in SUL or new 18F-FDGavid lesions that are typical of cancer and not related to treatment effect or infection $^{(18)}$.

\section{Re-Staging:}

For correct re-staging in monitoring the patients, 18FFDG PET/CT has several technological advantages over other

\section{REFERENCES}

1. Daniel V, Marcela A, Lorraine S, Axel L, Sylvie B \& C'ecile L : Role of computed tomography in the follow-up of hepatic and peritoneal metastases of GIST under imatinibmesylate treatment: a prospective study of 54 patients. European Journal of Radiology .54: 118-123; 2005.

2. Demetri GD, Benjamin RS, Blanke CD, et al.: NCCN Task Force report: management of patients with gastrointestinal stromal tumor (GIST) dupdate of the NCCN clinical practice guidelines. J Natl Compr Canc Netw.5 (Suppl 2): S1-29;2007.

3. Miettinen M, Sobin LH, Lasota J. Gastrointestinal stromal tumors of the diagnostic tests in GIST patients permit to detect small lesions using as a whole body technique which allow the diagnosis of disease in remote locations, Also, the use of integrated PET/CT scanners results in a correct anatomical localization of the hyper metabolic foci, substantially reducing false 17 positives $^{(19)}$. Results: Thus, PET/CT allows for maximum diagnostic capabilities, especially considering the follow up when metastatic lesions appear most often in unusual locations, especially at extra-abdominal site.

stomach: a clinicopathologic, immunohistochemical, and molecular genetic study of 1765 cases with long-term follow-up. Am J Surg Pathol .Jan. 29 (1): 52-68; 2005.

\section{Fong Y, Coit DG, Woodruff JM, et al.} Lymph node metastasis from soft tissue sarcoma in adults.Analysis of data from a prospective data- base of 1772 sarcoma patients. Ann Surg. 217:72-77; 1993.

5. Muna S, Robert C, Mary L \& Elaine K: Expression of Human Telomerase Reverse Transcriptase in Gastrointestinal Stromal Tumors Occurs Preferentially in Malignant Neoplasms. Hum Pathol. 35: 1231-1235; 2004. 
6. International union against cancer (UICC) Sobin LH, Wittekind Ch, editors. 7th ed. New York: Wiley. TNM classification of malignant tumors; 2010.

7. Fletcher CD, Berman JJ, Corless C, et al. Diagnosis of gastro- intestinal stromal tumors: a consensus approach. Hum Pathol . 33:459-465; 2002.

8. Demetri GD, von Mehren M, Antonescu CR, DeMatteo RP, Ganjoo KN, Maki RG, Pisters PW, Raut CP, Riedel RF, Schuetze S, Sundar HM, Trent JC, Wayne JD. NCCN Task Force report: update on the management of patients with gastrointestinal stromal tumors. J Natl Compr Canc Netw. 8(suppl 2):S1-S41. quiz S42-S44; 2010

9. Benjamin RS, Choi H, Macapinlac HA, et al. We should desist us- ing RECIST, at least in GIST. J Clin Oncol .25:17601764; 2007. Cancer Institute of the United States, National Cancer Institute of Canada. J Natl Cancer Inst.92:205-216; 2000

10. Isis Gayed, Thuan Vu et al. The Role of 18F-FDG PET in Staging and Early Prediction of Response to Therapy of Recurrent Gastrointestinal Stromal Tumors. J Nucl Med.45:17-21; 2004.

11. Choi H, Charnsangavej $\mathrm{C}$, de Castro Faria S, et al. CT evaluation of the response of gastrointestinal stromal tumors after imatinib mesylate treatment: a quantitative analysis correlated with FDG PET findings. Am J Roentgenol. 183:1619-1628; 2004.
12. Choi H, Faria SC, Benjamin RS, Podoloff DA, Macapinlac HA, Charnsangavej C. Monitoring treatment effects of STI-571 on gastrointestinal stromal tumors (GIST) with CT and PET: a quantitative analysis [abstract]. Radiology. 225: P583; 2002.

13. Gayed I, Vu T, Iyer R, Johnson M, Macapinlac H, Swanston N, Podoloff D. The role of 18F-FDG PET in staging and early prediction of response to therapy of recurrent gastrointestinal stromal tumors. J Nucl Med. 45:17-21; 2004.

14. Simó M, García JR, Soler M, Pérez G, López S, Lomeña FJ. Patterns of extension of gastrointestinal stromal tumors (GIST) treated with imatinib (Gleevec $\AA$ ) by 18F-FDG PET/CT .Rev Esp Med Nucl.25:80-8; 2006.

15. Goerres GW, Stupp R, Barghouth G, Hany TF, Pestalozzi B, Dizendorf E, et al. The value of PET, CT and in-line PET/CT in patients with gastrointestinal stromal tumours: long-term outcome of treatment with imatinib mesylate. Eur J Nucl Med Mol Imaging.32:153-62; 2005.

16. Otomi $\quad$ Y, Otsuka $\quad$ H, Morita N, Terazawa $\quad K$, Furutani $\quad K$, Harada M, Nishitani H. Relationship between FDG uptake and the pathological risk category in gastrointestinal stromal tumors. J Med Invest. 57:270-4; 2010.

17. Richard L. Wahl, Heather Jacene, Yvette Kasamon et al: From RECIST to 
PERCIST: Evolving Considerations for PET Response Criteria in Solid Tumors. J Nucl Med.50:122S-150S; 2009.

18. Eulalia Valls-Ferrusola, Juan Ramón García-Garzón et al: Patterns of extension of gastrointestinal stromal tumors (GIST) treated with imatinib (Gleevec) by 18F-FDG PET/CTPatterns of extension of gastrointestinal stromal tumors (GIST) treated with imatinib (Gleevec) by 18F-FDG PET/CT. Rev. esp. enferm. dig. 104; 2012. 
